PROCEEDINGS OF THE

AMERICAN MATHEMATICAL SOCIETY

Volume 129, Number 3, Pages 887-898

S 0002-9939(00)05756-7

Article electronically published on September 20, 2000

\title{
FREE ACTIONS OF $p$-GROUPS ON PRODUCTS OF LENS SPACES
}

\author{
ERGÜN YALÇIN
}

(Communicated by Ralph Cohen)

\begin{abstract}
Let $p$ be an odd prime number. We prove that if $(\mathbf{Z} / p)^{r}$ acts freely on a product of $k$ equidimensional lens spaces, then $r \leq k$. This settles a special case of a conjecture due to C. Allday. We also find further restrictions on non-abelian $p$-groups acting freely on a product of lens spaces. For actions inducing a trivial action on homology, we reach the following characterization: A $p$-group can act freely on a product of $k$ lens spaces with a trivial action on homology if and only if $\operatorname{rk}(G) \leq k$ and $G$ has the $\Omega$-extension property. The main technique is to study group extensions associated to free actions.
\end{abstract}

\section{INTRODUCTION}

The Brouwer fixed-point theorem states that any continuous map from the unit disc to itself has a fixed point. Thus $\mathbf{Z} / p$ cannot act freely on the unit disc. We also know by a classical result of $\mathrm{P}$. Smith that $\mathbf{Z} / p \times \mathbf{Z} / p$ cannot act freely on a sphere although $\mathbf{Z} / p$ can act freely on $S^{2 n-1}$ through complex multiplication. These results led to the concept of free rank of symmetry, defined as follows:

Definition 1.1. Let $X$ be a finite dimensional $C W$-complex, and $p$ be a prime number. The free $p$-rank of $X$, denoted by $\operatorname{frk}_{p}(X)$, is defined as the largest $r$ such that $(\mathbf{Z} / p)^{r}$ acts freely on $X$. The smallest value of $\operatorname{frk}_{p}(X)$ over all primes is called the free rank of $X$ and denoted by $\operatorname{frk}(X)$.

Free ranks of many interesting spaces are still not known. As a generalization of Smith's result it was conjectured that the free rank of a product of $k$ odd dimensional spheres is equal to $k$. For products of two spheres this was proved to be true by Heller 11. Later extending Gunnar Carlsson's results [9], 10] on homological trivial actions, A. Adem and W. Browder 4 proved that $\operatorname{frk}\left(\left(S^{n}\right)^{k}\right)=k$ when $n$ is an odd number different than 1, 3 or 7 . In [19, the free rank of $\left(S^{1}\right)^{k}$ is also proved to be $k$. For products of different dimensional spheres the problem of computing the free rank is still open.

Although free actions on products of real projective spaces are better understood, there are still many unanswered questions. In its general form, the problem of computing the free 2-rank of $\mathbf{R} P^{n_{1}} \times \cdots \times \mathbf{R} P^{n_{k}}$ is still open. For products of

Received by the editors May 12, 1999.

2000 Mathematics Subject Classification. Primary 57S25; Secondary 20J06, 20 D15.

Key words and phrases. Group actions, products of lens spaces, group extensions. 
equidimensional real projective spaces a complete solution to this problem is given in [5]. We found

$$
\operatorname{frk}_{2}\left(\left(\mathbf{R} P^{n}\right)^{k}\right)=\left\{\begin{aligned}
0, & \text { if } n \text { is even, } \\
k, & \text { if } n \equiv 1 \bmod 4, \\
2 k, & \text { if } n \equiv 3 \bmod 4 .
\end{aligned}\right.
$$

In this paper we study free actions on products of lens spaces. By a lens space, we mean the standard lens space $L_{p}^{2 n-1}\left(a_{1}, \ldots, a_{n}\right)$ where $\left(a_{i}, p\right)=1$, and we denote it simply by $L_{p}^{2 n-1}$. We prove the following:

Theorem A. If $(\mathbf{Z} / p)^{r}$ acts freely on a finite dimensional $C W$-complex homotopy equivalent to a product of $k$ equidimensional lens spaces, then $r \leq k$.

Since $(\mathbf{Z} / p)^{k}$ acts freely on $L_{p}^{2 n_{1}-1} \times \cdots \times L_{p}^{2 n_{k}-1}$ by a coordinatewise action, we obtain that

$$
\operatorname{frk}_{p}\left(L_{p}^{2 n_{1}-1} \times \cdots \times L_{p}^{2 n_{k}-1}\right)=k \quad \text { when } \quad n_{1}=\cdots=n_{k} .
$$

This proves the equidimensional case of a conjecture, due to C. Allday [6], which states that free $p$-rank of products of any $k$ lens spaces is $k$.

When the action on homology is a permutation action we prove:

Theorem B. If $G=(\mathbf{Z} / p)^{r}$ acts freely on a finite dimensional $C W$-complex $X$ homotopy equivalent to a product of $k$ equidimensional lens spaces by permuting a basis of $H_{1}\left(X, \mathbf{F}_{p}\right)$, then $r \leq \operatorname{dim}_{\mathbf{F}_{p}} H_{1}\left(X, \mathbf{F}_{p}\right)^{G}$.

We also show that if the lens spaces in the product are $(2 n-1)$-dimensional and $n$ is not a $p$ th power, then the action on homology is always a permutation action, and hence the stronger inequality holds in this case. We also note that Theorem B is analogous to a recent result of Adem and Benson [3] on free actions on products of equidimensional spheres.

In the rest of the paper, we study the conditions on a nonabelian $p$-group to act freely on a product of lens spaces with a trivial action on homology. There is a formal similarity between these conditions and the conditions on groups which can act freely on a torus. Recall that any finite group can act freely on some torus (see [13]), but when the action is required to induce a trivial action on homology in integral coefficients, only abelian groups can act freely. In particular the following is known (see [19]):

Theorem. A finite group $G$ can act freely on a $k$ torus with a trivial action on homology if and only if $\operatorname{rk}(G) \leq k$ and $G$ is abelian.

The cohomology of nonabelian $p$-groups which can act freely on a product of lens spaces resembles the cohomology of abelian $p$-groups in mod $p$ coefficients. There is a group theoretical explanation of such groups (due to T. Weigel [17]):

Definition. A group $G$ said to have $\Omega$-extension property if $G \cong P / \Omega_{1}(P)$ for some $p$-central $p$-group $P$ where $\Omega_{1}(P)=\left\langle x \in P \mid x^{p}=1\right\rangle$.

Recall that a group is called $p$-central if all the elements of order $p$ in the group are central. We prove the following:

Theorem C. A p-group $G$ can act freely on a product of $k$ lens spaces with a trivial action on homology if and only if $\operatorname{rk}(G) \leq k$ and $G$ has the $\Omega$-extension property. 
The free action that we construct comes from linear actions on spheres and hence the lens spaces in the product can be taken to have the same dimensions. This tells us that a $p$-group will act freely on a product of equidimensional lens spaces with a trivial action on homology if it acts freely on any product of lens spaces with a trivial action on homology. Note that the analogous statement for free $p$-group actions on products of spheres is not true. We discuss this further in Section 6

In section 5 we find another characterization of $p$-groups which have the $\Omega$ extension property in terms of an invariant called the Yagita invariant. It turns out that a $p$-group has the $\Omega$-extension property if and only if its Yagita invariant in $\bmod p$ coefficients is 2 . This gives a nice second characterization of $p$-groups that can act freely on products of lens spaces with a trivial action.

In the last section, we discuss some open problems about the free actions of $p$-groups on products of spheres and give some applications of the results of this paper to these problems.

Notation and conventions. Throughout the paper, $p$ denotes an odd prime number, and $\mathbf{Z} / p$ denotes integers modulo $p$. We call a group an elementary abelian $p$-group of rank $n$ if it is isomorphic to $(\mathbf{Z} / p)^{n}$, an $n$-fold product of $\mathbf{Z} / p$ with itself, for some integer $n$. By "rank of a $p$-group $G$ ", denoted by $\operatorname{rk}(G)$, we will always mean the rank of the largest elementary abelian $p$-subgroup, and the rank of a finite group will be the maximum value of the ranks of all its Sylow subgroups. Finally, when we are proving our results, we will assume that all the lens spaces $L_{p}^{2 n-1}$ have a dimension of at least 3 , because the results of this paper are already known to be true for products of $L_{p}^{1} \simeq S^{1}$ (see [19]).

\section{Proof of Theorem A}

Let $X$ be a finite $C W$-complex homotopy equivalent to a product of $k$ equidimensional lens spaces, which we denote by $X \simeq \prod_{k} L_{p}^{2 n-1}$, and let $G$ be a $p$-group acting freely on $X$. The covering map $X \rightarrow X / G$ gives an extension of $p$-groups

$$
0 \rightarrow \pi_{1}\left(X, x_{o}\right) \rightarrow \pi_{1}\left(X / G, \bar{x}_{o}\right) \rightarrow G \rightarrow 1
$$

where $\pi_{1}\left(X, x_{o}\right) \cong(\mathbf{Z} / p)^{k}$, and $P=\pi_{1}\left(X / G, \bar{x}_{o}\right)$ acts freely on the universal cover of $X$. Since the universal cover of $X$ is homotopy equivalent to $\left(S^{2 n-1}\right)^{k}$, we can apply the result of Adem and Browder [4] on group actions on products of spheres, and obtain that $\operatorname{rk}(P)=k$. So, to prove Theorem A, it is enough to prove the following statement:

Statement. If $0 \rightarrow(\mathbf{Z} / p)^{k} \rightarrow P \rightarrow(\mathbf{Z} / p)^{r} \rightarrow 1$ is an extension such that $\operatorname{rk}(P)=$ $k$, then $r \leq k$.

Unfortunately, this statement is not true. A counterexample to this statement (due to A. Caranti) was given in 6]. One can also find similar examples using the family of $p$-groups constructed by Ol'shanskii [14].

So, to prove Theorem A, one should look for other obstructions on the extensions associated with free actions. We prove the following:

Proposition 2.1. If a p-group $G$ acts freely on $X \simeq \prod_{k} L_{p}^{2 n-1}$, then as an $\mathbf{F}_{p} G$ module $\pi_{1}\left(X, x_{o}\right) \cong H_{2 n-1}\left(Y, \mathbf{F}_{p}\right)$ where $Y$ is the universal cover of $X$. In particular, as an $\mathbf{F}_{p} G$-module, $\pi_{1}\left(X, x_{o}\right)$ is a mod $p$ reduction of a $\mathbf{Z}$-free $\mathbf{Z} G$-module. 
Proof. Since $X \simeq \prod_{k} L_{p}^{2 n-1}$, there is a basis $x_{1}, \ldots, x_{k}$ for $H^{1}\left(X, \mathbf{F}_{p}\right)$ such that

$$
H^{*}\left(X, \mathbf{F}_{p}\right) \cong \bigwedge_{\mathbf{F}_{p}}\left(x_{1}, \ldots, x_{k}\right) \otimes \mathbf{F}_{p}\left[\beta x_{1}, \ldots, \beta x_{k}\right] /\left(\left(\beta x_{1}\right)^{n}, \ldots,\left(\beta x_{k}\right)^{n}\right) .
$$

We first observe the following:

Lemma 2.2. If $n$ is not a pth power, then $G$ must permute the $x_{i}$ 's.

Proof. This is because of the Binomial Coefficient Theorem. Observe that since the $\mathbf{F}_{p}$-vector space generated by $\left(\beta x_{i}\right)^{n}$ 's is an $\mathbf{F}_{p} G$-module, for any $j, g\left(\beta x_{j}\right)^{n}$ must be a linear combination of $\left(\beta x_{i}\right)^{n}$ 's. If $n$ is not a $p$ th power, then $g\left(\beta x_{j}\right)^{n}=$ $\left(g \beta x_{j}\right)^{n}=\left(\sum a_{i} \beta x_{i}\right)^{n}$ will not be spanned by $\left(\beta x_{1}\right)^{n}, \ldots,\left(\beta x_{k}\right)^{n}$ unless all $a_{i}$ 's except one are zero.

Let $V$ be the vector space generated by $\left(\beta x_{i}\right)^{n}$ 's. The above lemma in particular tells us that when $n$ is not a $p$ th power, $H^{1}\left(X, \mathbf{F}_{p}\right) \cong V$ as an $\mathbf{F}_{p} G$-module. Observe that when $n$ is a $p$ th power, this is still true since the $p$ th power map gives an isomorphism between $\mathbf{F}_{p} G$-modules generated by $x_{i}$ 's and $\mathbf{F}_{p} G$-modules generated by $x_{i}^{p}$ 's. So, $H^{1}\left(X, \mathbf{F}_{p}\right) \cong V$ for all $n$.

Now, consider the $E=\pi_{1}\left(X, x_{o}\right)$ action on $Y$. The Cartan-Leray spectral sequence for this action gives the following short exact sequence of $G$-modules:

$$
0 \rightarrow H^{2 n-1}\left(Y, \mathbf{F}_{p}\right) \stackrel{d_{2 n}}{\longrightarrow} H^{2 n}\left(E, \mathbf{F}_{p}\right) \stackrel{\pi^{*}}{\longrightarrow} H^{2 n}\left(X, \mathbf{F}_{p}\right) \rightarrow 0 .
$$

Since ker $\pi^{*}=V \cong H^{1}\left(X, \mathbf{F}_{p}\right)$, we obtain that $H^{1}\left(X, \mathbf{F}_{p}\right) \cong H^{2 n-1}\left(Y, \mathbf{F}_{p}\right)$ as $\mathbf{F}_{p} G$-modules. Taking duals, we conclude $\pi_{1}\left(X, x_{o}\right) \cong H_{2 n-1}\left(Y, \mathbf{F}_{p}\right)$.

Now, we prove a result on group extensions to complete the proof of Theorem A.

Proposition 2.3. Let $0 \rightarrow E \rightarrow P \rightarrow G \rightarrow 1$ be an extension where $E \cong(\mathbf{Z} / p)^{k}$, $G \cong(\mathbf{Z} / p)^{r}$ and $\operatorname{rk}(P)=k$. Suppose that as an $\mathbf{F}_{p} G$-module $E$ is isomorphic to the $\bmod p$ reduction of a $\mathbf{Z}$-free $\mathbf{Z} G$-module. Then, $r \leq k$.

Proof. Let $M$ be a $\mathbf{Z}$-free $\mathbf{Z} G$-module such that $E \cong \mathbf{F}_{p} \otimes M$ as an $\mathbf{F}_{p} G$-module. Let $H$ be the subgroup of elements which acts trivially on $M$. Then, $G / H$ acts faithfully on $M$, and by a result of A. Adem and W. Browder in [4, we have

$$
\operatorname{rk}(G / H) \leq \frac{1}{p-2}\left(k-\operatorname{rk}\left(E^{G}\right)\right) \leq k-\operatorname{rk}\left(E^{G}\right)
$$

where $E^{G}$ denotes the invariant submodule of $E$ under $G$ action. So, to prove the lemma it is enough to show that $\operatorname{rk}(H) \leq \operatorname{rk}\left(E^{G}\right)$. We will do this by constructing an injective homomorphism from $H$ to $E^{G}$.

Let $\Phi: H \rightarrow E$ be the map defined as $\Phi(h)=x^{p}$ where $x$ is a representative of $h$ in $P$. Since $H$ acts trivially on $E, \Phi(h)$ is independent from the choice of $x$. Moreover, for any $x, y \in G$ which represents $h_{1}$ and $h_{2}$ in $H$, we have $(x y)^{p}=$ $x^{p} y^{p}[y, x]^{p(p-1) / 2}=x^{p} y^{p}$, which implies that $\Phi\left(h_{1} h_{2}\right)=\Phi\left(h_{1}\right) \Phi\left(h_{2}\right)$. Hence, $\Phi$ is a homomorphism. Moreover, since $E$ is a maximal elementary abelian subgroup, $\Phi(h)$ cannot be zero for any $h \in H$, so, $\Phi$ is injective. Finally, let $g \in G$ and $h \in H$. If $x \in G$ is an element representing $h$, then we have $[x,[x, g]]=1$ which implies $\left[x^{p}, g\right]=[x, g]^{p}=1$. This shows that $g$ acts trivially on $\Phi(h)=x^{p}$. Since this is true for all $g \in G$ and $h \in H$, we obtain $\Phi(H) \subseteq E^{G}$. This completes the proof.

Thus, the proof of Theorem A is complete. 


\section{Proof of Theorem B}

In the previous section we observed that when a $p$-group $G$ acts freely on a finite complex $X$ homotopy equivalent to a product of $k$ equidimensional lens spaces, i.e. $X \simeq \prod_{k} L_{p}^{2 n-1}$, there exists an extension of the form

$$
0 \rightarrow E \rightarrow P \rightarrow G \rightarrow 1
$$

where $E \cong(\mathbf{Z} / p)^{k}$ and $\operatorname{rk}(P)=k$. We also showed that $G$ action on $E$ comes from a $\mathbf{Z}$-free $\mathbf{Z} G$-module. In this section we will study the situation where $G$ action on $E$ is a permutation action. For this we need a stronger condition for the extension which is defined as follows:

Definition 3.1. Let $G$ be a finite group, and let $M$ be a $G$-module. A class $\alpha \in H^{n}(G, M)$ is called special if $\operatorname{res}_{C}^{G} \alpha \neq 0$ for every cyclic subgroup $C \subseteq G$ of prime order. An extension $0 \rightarrow M \rightarrow P \rightarrow G \rightarrow 1$ is called special if the corresponding extension class $\alpha \in H^{2}(G, M)$ is special.

We prove that in the given situation the associated extension is special.

Proposition 3.2. If a p-group $G$ acts freely on $X \simeq \prod_{k} L_{p}^{2 n-1}$, and if every element of order $p$ in $G$ acts on homology by permuting a basis, then $0 \rightarrow \pi_{1}\left(X, x_{o}\right) \rightarrow$ $\pi_{1}\left(X / G, \bar{x}_{o}\right) \rightarrow G \rightarrow 1$ is a special extension.

Proof. First observe that the restriction of the given extension $0 \rightarrow \pi_{1}\left(X, x_{o}\right) \rightarrow$ $\pi_{1}\left(X / G, \bar{x}_{o}\right) \rightarrow G \rightarrow 1$ to a cyclic subgroup $C$ of order $p$ is equivalent as extensions to the associated extension

$$
0 \rightarrow \pi_{1}\left(X, x_{o}\right) \rightarrow \pi_{1}\left(X / C, \bar{x}_{i}\right) \rightarrow C \rightarrow 1
$$

of the reduced $C$ action on $X$. So, it is enough to prove the proposition for the extensions associated to free $\mathbf{Z} / p$ actions. To the contrary, assume that $G=\mathbf{Z} / p$ acts freely on $X$ and the associated extension splits. We will reach a contradiction.

Consider the following lemma, due to A. Adem [1]:

Lemma 3.3. $G \cong \mathbf{Z} / p, X$ is a finite dimensional $G$-space with

$$
H^{i}\left(X, \mathbf{F}_{p}\right) \cong\left\{\begin{array}{cc}
\mathbf{F}_{p}, & i=N \\
0, & i>N
\end{array}\right.
$$

If the generator $\mu \in H^{N}\left(X, \mathbf{F}_{p}\right)$ is in the image of $i^{*}$, where $i: X \rightarrow E G \times_{G} X$ is the fiber inclusion, then the action has fixed points.

Since $X \simeq \prod_{k} L_{p}^{2 n-1}$ satisfies the conditions of this lemma, the image of $i^{*}$ on the top dimension must be zero because $G \cong \mathbf{Z} / p$ acts freely on $X$.

On the other hand, the map $i: X \rightarrow E G \times_{G} X \simeq X / G$ can be written as the quotient map $Y / E \rightarrow Y / P$ where $Y$ is the universal cover of $X, E=\pi_{1}\left(X, x_{o}\right)$, and $P=\pi_{1}\left(X / G, \bar{x}_{o}\right)$. So, it fits into the following commutative diagram:

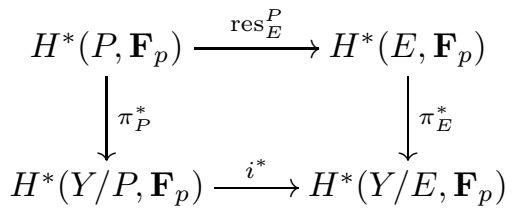

where the vertical maps come from the projections $\pi_{P}: Y / P \simeq E P \times_{P} Y \rightarrow B P$ and $\pi_{E}: Y / E \rightarrow B E$. Since $0 \rightarrow E \rightarrow P \rightarrow G \rightarrow 1$ is a split extension, the image 
of the restriction map $\operatorname{res}_{E}^{P}$ is exactly $H^{*}\left(E, \mathbf{F}_{p}\right)^{G}$, the subring of the invariant classes. So, if we can show that $\pi_{E}^{*}(u) \neq 0$ for some $u \in H^{N}\left(E, \mathbf{F}_{p}\right)^{G}$ where $N$ is the top dimension, then by the commutativity of the diagram, the image of $i^{*}$ will be nontrivial, and hence we will reach a contradiction. So, to complete the proof it is enough to show that there is a class $u$ in $H^{*}\left(E, \mathbf{F}_{p}\right)^{G}$ such that $\pi_{E}^{*}(u) \neq 0$.

Let $x_{1}, \ldots, x_{k}$ be a basis for $H^{1}\left(E, \mathbf{F}_{p}\right)$ such that ker $\pi_{E}$ is generated by $\left(\beta x_{i}\right)^{n}$ 's. If $n$ is not a $p$ th power, then $G$ must permute $x_{i}$ 's; hence we can take $u=$ $\prod_{i=1}^{k} x_{i}\left(\beta x_{i}\right)^{n-1}$ as the desired class. So, assume $n$ is a $p$ th power. In this case, we have

$$
H^{*}\left(X, \mathbf{F}_{p}\right) \cong \bigwedge_{\mathbf{F}_{p}}\left(t_{1}, \ldots, t_{k}\right) \otimes \mathbf{F}_{p}\left[\beta t_{1}, \ldots, \beta t_{k}\right] /\left(\left(\beta t_{1}\right)^{n}, \ldots,\left(\beta t_{k}\right)^{n}\right)
$$

for any basis chosen in $H^{1}\left(E, \mathbf{F}_{p}\right)$. Let $t_{1}, \ldots, t_{k}$ be a basis permuted by $G$. Then, the product $u=\prod_{i=1}^{k} t_{i}\left(\beta t_{i}\right)^{n-1}$ is an element in $H^{*}\left(E, \mathbf{F}_{p}\right)^{G}$, and $\pi_{E}^{*}(u) \neq 0$. The proof is complete.

Now, the proof of Theorem B follows from the following result:

Proposition 3.4. Suppose that $0 \rightarrow E \rightarrow P \rightarrow G \rightarrow 1$ is a special extension where $E \cong(\mathbf{Z} / p)^{k}, G \cong(\mathbf{Z} / p)^{r}$, and $G$ acts on $E$ by permuting a basis. Then $r \leq \operatorname{rk}\left(E^{G}\right)$.

To prove this we need the following lemma:

Lemma 3.5. Let $G \cong(\mathbf{Z} / p)^{r}$ and $M \cong \mathbf{F}_{p}[G / H]$ as an $\mathbf{F}_{p} G$-module for some $H \subseteq G$. Then, for every $\alpha \in H^{2}(G, M)$, there exists an index $p$ subgroup $G_{\alpha} \subseteq G$ such that $\operatorname{res}_{\langle g\rangle}^{G} \alpha=0$ for every $g \in G_{\alpha}$.

Proof. Let $0 \rightarrow M \rightarrow P \rightarrow G \rightarrow 1$ be an extension represented by $\alpha$. Since $H \subseteq G$ acts trivially on $M$, we can define a homomorphism $\Phi: H \rightarrow M^{G}$ as in the proof of Lemma 2.3. Since $M^{G} \cong \mathbf{F}_{p}$, ker $\Phi$ is an index $p$ subgroup of $H$. Let $K \subseteq G$ such that $G \cong H \times K$, and let $G_{\alpha}=(\operatorname{ker} \Phi) K$. For any $g \in G_{\alpha}$, we have two possibilities: Assume $g \in \operatorname{ker} \Phi$, then $\Phi(g)=0$; hence the extension splits when restricted to $\langle g\rangle$. By the theory of group extensions, this means $\operatorname{res}_{\langle g\rangle}^{G} \alpha=0$. Now, assume $g \notin \operatorname{ker} \Phi$, then $g$ is not in $H$, and hence it acts by permuting a basis. Since $H^{*}\left(\langle g\rangle, \mathbf{F}_{p}[\langle g\rangle]\right)=0, \operatorname{res}_{\langle g\rangle}^{G} \alpha$ is zero. So, $G_{\alpha}$ is a subgroup with the desired properties.

Proof of Proposition 3.4. Since $G$ action on $E$ is a permutation action, as an $\mathbf{F}_{p} G$ module, $E \cong \bigoplus_{i=1}^{l} \mathbf{F}_{p}\left[G / H_{i}\right]$ for some subgroups $H_{i} \subseteq G$, and the extension class $\alpha$ can be written as $\left(\alpha_{1}, \ldots, \alpha_{l}\right) \in H^{2}(G, E) \cong \bigoplus_{i=1}^{l} H^{2}\left(G, \mathbf{F}_{p}\left[G / H_{i}\right]\right)$. By the above lemma, for every $\alpha_{i}$ we can find an index $p$ subgroup $G_{i} \subseteq G$ such that restriction of $\alpha_{i}$ to any cyclic subgroup of $G_{i}$ is zero. Note that the intersection of all $G_{i}$ 's must be trivial, because otherwise there will be a cyclic subgroup $C$ where $\operatorname{res}_{C}^{G} \alpha_{i}=0$ for all $i$, and this will contradict the speciality of $\alpha$. Since the intersection with an index $p$ subgroup decreases the rank by 1 , to have a trivial intersection, we must have $r \leq l=\operatorname{rk}\left(E^{G}\right)$.

Remark 3.6. It is interesting to ask if Proposition 3.4 is still true without the assumption that $G$ acts on $E$ by permuting a basis. The answer is no. To demonstrate this, we consider the $p$-group $P$ generated by $s$ and $s_{1}$ with the relations

$$
s_{2}=\left[s_{1}, s\right], s_{3}=\left[s_{2}, s\right], 1=\left[s_{3}, s\right], s^{3}=s_{1}^{-3}=s_{3},
$$


and

$$
\left[s_{i}, s_{j}\right]=s_{2}^{3}=s_{3}^{3}=1 \text { for all } i \in\{1,2,3\} .
$$

The basic properties of this group are explained in [15. In particular, $P^{\prime}=\Omega_{1}(P)=$ $\left\langle s_{2}, s_{3}\right\rangle$, and $P^{\prime}$ and $P / P^{\prime}$ are both elementary abelian groups of rank 2. Taking $E=P^{\prime}$ and $G=P / E$, we obtain a special extension $0 \rightarrow E \rightarrow P \rightarrow G \rightarrow 1$ where $E \cong(\mathbf{Z} / p)^{2}$ and $G \cong(\mathbf{Z} / p)^{2}$. But, the conclusion of Proposition 3.4 is not true for this extension, $\operatorname{since} \operatorname{rk}(G)=2>\operatorname{rk}\left(E^{G}\right)=1$.

We conclude this section with a corollary of Theorem B:

Corollary 3.7. If $G=(\mathbf{Z} / p)^{r}$ acts freely on a finite dimensional $C W$-complex $X \simeq \prod_{k} L_{p}^{2 n-1}$ where $n$ is not a pth power, then $r \leq \operatorname{dim}_{\mathbf{F}_{p}} H_{1}\left(X, \mathbf{F}_{p}\right)^{G}$.

Proof. This follows from Lemma 2.2 and Theorem B.

\section{Proof of Theorem C}

We start with a lemma:

Lemma 4.1. If $G$ acts freely on a finite dimensional $C W$-complex $X \simeq L_{p}^{2 n_{1}-1} \times$ $\cdots \times L_{p}^{2 n_{k}-1}$ with a trivial action on homology, then the associated extension $0 \rightarrow$ $\pi_{1}\left(X, x_{o}\right) \rightarrow \pi_{1}\left(X / G, \bar{x}_{o}\right) \rightarrow G \rightarrow 1$ is special.

Proof. In the case of trivial action on homology, one can easily modify the proof of Proposition 3.2 so that it works for products of different dimensional lens spaces.

Now, we are ready to prove Theorem C.

Proof of Theorem C. $(\Rightarrow)$ Let $G$ be a $p$-group acting freely on $X \simeq L_{p}^{2 n_{1}-1} \times \cdots \times$ $L_{p}^{2 n_{k}-1}$ with trivial action on homology. Let $E=\pi_{1}\left(X, x_{o}\right)$ and $P=\pi_{1}\left(X / G, \bar{x}_{o}\right)$. By the above lemma, the associated extension $0 \rightarrow E \rightarrow P \rightarrow G \rightarrow 1$ is special. This implies that elements of order $p$ in $P$ are included in $E$. So, $E=\Omega_{1}(P)=$ $\left\langle g \in P \mid g^{p}=1\right\rangle$. Note also that $E$ is a central subgroup of $P$ because the action of $G$ on homology is trivial. So, $P$ is $p$-central with $G \cong P / \Omega_{1}(P)$, i.e. $G$ has the $\Omega$ extension property (see the introduction for definition). The inequality $\operatorname{rk}(G) \leq k$ follows from Proposition 2.3 or Proposition 3.4 .

$(\Leftarrow)$ Assume that $G$ is a $p$-group with $\operatorname{rk}(G) \leq k$, and it has the $\Omega$-extension property. Then, there is a $p$-central $p$-group $P$ such that $G \cong P / \Omega_{1}(P)$, and moreover, we can assume that $\operatorname{rk}(P)=\operatorname{rk}(G)$ (see Proposition 4.1 (d) and Remark 5.3 in [16]). Let $E=\Omega_{1}(P)$ and $t=\operatorname{rk}(E)$. Choose a set of generators $e_{1}, \ldots, e_{t}$ for $E$, and let $H_{j}$ be the subgroup generated by $e_{i}$ 's with $i \neq j$. For each $i, H_{i}$ is central in $P$, and $e_{i}$ is central in $P / H_{i}$. Take the regular one dimensional complex representation of $\left\langle e_{i}\right\rangle$ and induce it to $P / H_{i}$. The resulting representation is a linear action on $|G|$ dimensional complex vector space. Let $S(i)$ be the unit sphere of the underlying $2|G|$ dimensional Euclidean space. Then, $P$ acts on $S(i)$ through the quotient map $P \rightarrow P / H_{i}$, and it is easy to see that every element in the difference set $E-H_{i}$ acts freely on $S(i)$.

Now, consider the diagonal action of $P$ on the product $X=S(1) \times \cdots \times S(t)$. Since the difference sets $E-H_{i}$ 's cover $E$, the elements of $E$ act on the product without fixed points. Note that any element in $P$ has a power that lies in $E$. So, the elements of $P$ cannot have fixed points either; hence $P$ acts freely on $X$. Consider the induced free action of $G \cong P / E$ on $X / E$. It is easy to see that $X / E \simeq\left(L_{p}^{2 n-1}\right)^{t}$ 
with $n=|G|$ and $t \leq k$. By adding more lens spaces (with trivial $G$ action) to the product, one gets a free $G$ action on a product of $k$ lens spaces. Clearly, this action induces a trivial action on homology.

Observe that the above construction gives us a free action on a product of equidimensional lens spaces. This leads to the following corollary:

Corollary 4.2. If a p-group $G$ acts freely on a product of $k$ lens spaces with a trivial action on homology, then it can act freely on a product of $k$ equidimensional lens spaces with a trivial action on homology.

This is an interesting outcome, since the analogous statement for free actions of $p$-groups on products of spheres is not true. To see this, consider the non-abelian $p$-group $G$ of order $p^{3}$ and exponent $p$. G. Lewis [12] and K. Alzubaidy 7] showed that $G$ cannot act freely on $S^{n} \times S^{n}$ for any $n$. On the other hand, a recent result of A. Adem and J. Smith [2] shows that $G$ acts freely on a finite complex homotopy equivalent to a product of two spheres.

\section{YAGITA'S INVARIANT}

In this section we will study Yagita's invariant, and its relations to group extensions. The Yagita invariant in $\bmod p$ coefficients is defined as follows:

Let $G$ be a finite group. For any imbedding $i: \mathbf{Z} / p \hookrightarrow G$, there exists a maximum number $m$ such that the image under restriction $i^{*}: H^{*}\left(G, \mathbf{F}_{p}\right) / \sqrt{0} \rightarrow$ $H^{*}\left(\mathbf{Z} / p, \mathbf{F}_{p}\right) / \sqrt{0}$ is contained in $\mathbf{F}_{p}\left[u^{m}\right]$, where $u$ is a generator of $H^{2}\left(\mathbf{Z} / p, \mathbf{F}_{p}\right)$. The Yagita invariant of $G$ is defined as the least common multiple of the values $2 m$ for all inclusions $\mathbf{Z} / p \hookrightarrow G$, and it is denoted by $\operatorname{po}(G, \mathbf{Z} / p)$. The following are the known properties of $\operatorname{po}(G, \mathbf{Z} / p)$ (see [18] for proofs):

i) If $G$ is abelian, then $\operatorname{po}(G, \mathbf{Z} / p)=2$.

ii) If $G$ is a $p$-group, then $\operatorname{po}(G, \mathbf{Z} / p)=2 p^{s}$ for some $s \geq 0$.

iii) If $H \subseteq G$, then $\operatorname{po}(H, \mathbf{Z} / p)$ divides $\operatorname{po}(G, \mathbf{Z} / p)$.

By computing the Yagita invariant of minimal nonabelian groups, Yagita proved the following:

Proposition 5.1 (Yagita). If $G$ is a p-group which contains an order $p$ element outside the center, then $2 p$ divides $\operatorname{po}(G, \mathbf{Z} / p)$.

This shows that $p$-groups with $\operatorname{po}(G, \mathbf{Z} / p)=2$ must be $p$-central, but the converse is not true in general. Being a stronger condition, $\Omega$-extension property provides a characterization for $p$-groups with po $(G, \mathbf{Z} / p)=2$. We prove the following:

Proposition 5.2. Let $G$ be a p-group. Then, $G$ has the $\Omega$-extension property if and only if the Yagita invariant of $G$ in $\bmod p$ coefficients is 2 .

For the proof of this proposition, we need the following lemma:

Lemma 5.3. Let $G$ be a p-group and let $g$ be a central element of order $p$ in $G$. Suppose that

$$
\operatorname{res}_{\langle g\rangle}^{G}: H^{2}\left(G, \mathbf{F}_{p}\right) \rightarrow H^{2}\left(\langle g\rangle, \mathbf{F}_{p}\right)
$$

is a zero map; then $2 p$ divides $\operatorname{po}(G, \mathbf{Z} / p)$. 
Proof. Consider the Hochschild-Serre spectral sequence

$$
H^{*}\left(G /\langle g\rangle, H^{*}\left(\langle g\rangle, \mathbf{F}_{p}\right)\right) \Rightarrow H^{*}\left(G, \mathbf{F}_{p}\right)
$$

associated to the extension $1 \rightarrow\langle g\rangle \rightarrow G \rightarrow G /\langle g\rangle \rightarrow 1$. Let $u \in H^{2}\left(\langle g\rangle, \mathbf{F}_{p}\right)$ be a nonzero class. Since $\operatorname{res}_{\langle g\rangle}^{G}$ is a zero map on dimension two, $d_{3}(u) \neq 0$. Then, for all $u^{m}$ where $p$ does not divide $m, d_{3}\left(u^{m}\right)=m d_{3}(u) u^{m-1} \neq 0$. This implies that $\operatorname{im}\left\{\operatorname{res}_{\langle g\rangle}^{G}: H^{*}\left(G, \mathbf{F}_{p}\right) \rightarrow H^{*}\left(\langle g\rangle, \mathbf{F}_{p}\right)\right\}$ is in $\mathbf{F}_{p}\left[u^{p}\right]$. Hence, $2 p$ divides $\operatorname{po}(G, \mathbf{Z} / p)$.

Proof of Proposition 5.2. $(\Rightarrow)$ Assume that $G$ has the $\Omega$-extension property; then $G$ fits into a central extension

$$
0 \rightarrow E \rightarrow P \rightarrow G \rightarrow 1
$$

where $E$ is the maximal elementary abelian subgroup of $p$-central group $P$. Let $\alpha \in H^{2}(G, E)$ be the class representing this extension. Since for any element $g \in G$ of order $p$, the restriction of $(*)$ to the cyclic subgroup generated by $g$ does not split, $\operatorname{res}_{\langle g\rangle}^{G} \alpha$ cannot be zero. Hence, $\operatorname{po}(G, \mathbf{Z} / p)=2$.

$(\Leftarrow)$ Assume $G$ is a $p$-group with $\operatorname{po}(G, \mathbf{Z} / p)=2$. By Proposition 5.1 is $p$-central, i.e. every element of order $p$ in $G$ is central. Let $g \in G$ be an element of order $p$. Since $\operatorname{po}(G, \mathbf{Z} / p)=2$ and $g$ is central, we can apply Lemma 5.3, and obtain that $\operatorname{res}_{\langle g\rangle}^{G}: H^{2}\left(G, \mathbf{F}_{p}\right) \rightarrow H^{2}\left(\langle g\rangle, \mathbf{F}_{p}\right)$ is a nonzero map. Let $x_{g} \in H^{2}\left(G, \mathbf{F}_{p}\right)$ be an element such that $\operatorname{res}_{\langle g\rangle}^{G} x_{g} \neq 0$. Let $\alpha \in H^{2}\left(G,(\mathbf{Z} / p)^{t}\right)$ be the $t$-tuple of elements $x_{g}$ for each $g \in G$ of order $p$, and let $1 \rightarrow(\mathbf{Z} / p)^{t} \rightarrow P \rightarrow G \rightarrow 1$ be an extension corresponding to the class $\alpha$. Since $(\mathbf{Z} / p)^{t}$ was taken as a trivial $G$-module, this extension is central. Moreover, it is special because $\operatorname{res}_{\langle g\rangle}^{G} \alpha \neq 0$. This implies that $P$ is $p$-central with $\Omega_{1}(P)=(\mathbf{Z} / p)^{t}$. Hence, $G$ has the $\Omega$-extension property.

We have the following immediate corollary to Proposition 5.2

Corollary 5.4. A p-group $G$ can act freely on a product of $k$ lens spaces with a trivial action on homology if and only if $\operatorname{rk}(G) \leq k$ and $\operatorname{po}(G, \mathbf{Z} / p)=2$.

\section{FreE ACtions of $p$-Groups on PRODUCTS OF SPHERES}

The main question that we are interested in is the following:

Question 6.1. Is there a non-abelian $p$-group of rank $r$ and exponent $p$ which can act freely on a product of $r$ equidimensional spheres?

In particular we will consider the extra-special $p$-groups of exponent $p$. The smallest such group is $G_{p^{3}}$, the nonabelian $p$-group of order $p^{3}$ and exponent $p$. $G_{p^{3}}$ has rank 2, but by the results of G. Lewis [12] and K. Alzubaidy 7] we know:

Theorem 6.2 (Lewis, Alzubaidy). $G_{p^{3}}$ cannot act freely on $S^{n} \times S^{n}$ for any $n$.

Although there is little evidence, one may try to show that the other extra-special $p$ groups of exponent $p$ cannot act freely on $\left(S^{n}\right)^{r}$ where $r$ is the rank of the group. As a preliminary study, we find restrictions on the the orbit spaces of such actions using the results of the previous sections.

Proposition 6.3. Assume $p$ is an odd prime such that $p>3$. Let $G$ be a p-group of rank $r$ and $G_{r}$ be the subgroup generated by centralizers of elementary abelian subgroups of rank $r$. If $G$ acts freely on $X \simeq\left(S^{n}\right)^{r}$, then $G_{r}$ acts trivially on 
homology. In particular, if $G$ is an extra-special p-group of exponent $p$, or $G$ is p-group where $H^{*}(G)$ is Cohen-Macaulay, then $G$ acts trivially on homology.

Proof. Let $E \subseteq G$ be an elementary abelian subgroup of rank $r$. Since $E$, act freely on $X$, by a result of Adem and Browder [4, we have

$r=\operatorname{rk}(E) \leq \operatorname{dim}_{\mathbf{F}_{p}} H_{n}\left(X, \mathbf{F}_{p}\right)^{E}+\frac{1}{p-2}\left(\operatorname{dim}_{\mathbf{F}_{p}} H_{n}\left(X, \mathbf{F}_{p}\right)-\operatorname{dim}_{\mathbf{F}_{p}} H_{n}\left(X, \mathbf{F}_{p}\right)^{E}\right)$.

Since the dimension of $H_{n}\left(X, \mathbf{F}_{p}\right)$ is also $r$, for the inequality to hold, $E$ must act trivially on $H_{n}\left(X, \mathbf{F}_{p}\right)$. When the action on homology is trivial we have the following exact sequence associated to a free action on $X$ :

$$
0 \rightarrow H^{n}\left(X, \mathbf{F}_{p}\right) \stackrel{d_{n+1}}{\longrightarrow} H^{n+1}\left(E, \mathbf{F}_{p}\right) \longrightarrow H^{n+1}\left(X / E, \mathbf{F}_{p}\right)
$$

Since the maps of this sequence are $G$ maps, any element acting trivially on $E$ should act trivially on the homology of $X$. Therefore the centralizers of $E$, and hence $G_{r}$, act trivially on $H_{n}\left(X, \mathbf{F}_{p}\right)$. The last statement of the proposition follows from the fact that for the groups given, $G=G_{r}$ (see [8]).

Proposition 6.4. Let $G$ be a p-group of rank $r$ with $p>3$. If $G$ acts freely on $X \simeq\left(S^{2 n-1}\right)^{r}$ such that $X / E \simeq \prod_{r} L_{p}^{2 n-1}$ for some $E \cong(\mathbf{Z} / p)^{r} \subseteq G$, then $E$ is the unique elementary abelian subgroup of rank $r$.

Proof. Let $N$ be the normalizer of $E$ in $G_{r}$. Applying Proposition 2.1 to the $N / E$ action on $X / E \simeq \prod_{r} L_{p}^{2 n-1}$, we obtain that as an $\mathbf{F}_{p}(N / E)$-module,

$$
E=\pi_{1}\left(X / E, x_{0}\right) \cong H_{n}\left(X, \mathbf{F}_{p}\right) .
$$

On the other hand, $G_{r}$ acts trivially on $H_{n}\left(X, \mathbf{F}_{p}\right)$ by the above proposition, so $N$ acts trivially on $E$. This implies that the normalizer of $E$ in $G_{r}$ is the same as the centralizer of $E$ in $G_{r}$, but in the case of a $p$-group this is only possible when they both are equal to the entire group $G_{r}$ (see Lemma 2.3 in [17). So, $E$ is central in $G_{r}$, and hence $E$ is the unique elementary abelian subgroup of rank $r$ in $G$.

From these we conclude:

Corollary 6.5. If an extra-special p-group $G$ of exponent $p$ acts freely on $X \simeq$ $\left(S^{n}\right)^{\mathrm{rk}(G)}$, then $G$ acts trivially on homology, and $X / E$ is not homotopy equivalent to a product of lens spaces for any maximal elementary abelian subgroup $E \subseteq G$.

Corollary 6.6. For a p-group of rank $r$ with $p>3$, the following are equivalent:

i) $H^{*}(G)$ is Cohen-Macaulay and $G$ acts freely on $X \simeq\left(S^{n}\right)^{r}$ where $X / E \simeq$ $\prod_{r} L_{p}^{2 n-1}$ for some maximal elementary abelian subgroup $E$,

ii) $G$ is p-central.

Now, we construct some examples:

Example 6.7. This example shows that $p$-groups which attain free actions as in Proposition 6.4 do not have to be $p$-central. Let

$$
\left.G=\left\langle a, b_{1}, \ldots, b_{p}\right| a^{p^{2}}=b_{i}^{p}=1, b_{i}^{a}=b_{i-1} \text { for all } i>1, b_{1}^{a}=b_{p}\right\rangle,
$$

and let $X=\left(S^{3}\right)^{p+1}$. Considering $S^{3} \subseteq \mathbf{C}^{2}$, we define a $G$ action on $X$ as follows:

$$
\begin{gathered}
a\left(x_{1}, \ldots, x_{p}, y\right)=\left(x_{p}, x_{1}, \ldots, x_{p-1}, \xi y\right), \\
b_{i}\left(x_{1}, \ldots, x_{p}, y\right)=\left(x_{1}, \ldots, \sigma x_{i}, \ldots, x_{p}, y\right)
\end{gathered}
$$


for all $i=1, \ldots, p$ where $\sigma$ is the $p$ th root of unity and $\xi$ is the $p^{2}$ th root of unity. It is clear from the construction that the action is free and nontrivial on homology and if $E=\left\langle a^{p}, b_{1}, \ldots, b_{p}\right\rangle$, then $X / E \simeq\left(L_{p}^{3}\right)^{p+1}$. Here $G_{r}=E$ and $E$ is the unique maximal elementary abelian subgroup, and it is not central.

Example 6.8. This example shows that the converse of Proposition 6.4 is not true in general. Let $G=\left\langle a, b \mid a^{p^{2}}=b^{p}=1, b^{-1} a b=a^{1+p}\right\rangle$. Note that $G$ has a unique maximal elementary abelian subgroup $E=\left\langle a^{p}, b\right\rangle$. We claim that $G$ cannot act freely on $X \simeq S^{n} \times S^{n}$ where $n$ is odd, with $X / E \simeq L_{p}^{n} \times L_{p}^{n}$. First observe that if $G$ acts freely on $S^{n} \times S^{n}$, then because of the Lefschetz fixed-point theorem, it should act trivially on homology. If $X / E$ is a product of two lens spaces, then $G$ must also act trivially on $\pi_{1}(X / E)=E$. However, $G$ acts nontrivially on $E$.

Another interesting discussion concerns the dimensions of the spheres in a product which admits a free action of a $p$-group. Yagita showed that if a $p$-group acts freely on $\left(S^{n}\right)^{k}$ with a trivial action on homology where $n+1$ is not divisible by $p$, then $G$ must be $p$-central. He proved this by showing that if a $p$-group $G$ acts freely on $\left(S^{n}\right)^{k}$ with a trivial action on homology, then the Yagita invariant of $G$ must divide $n+1$. The results of the previous section extend Yagita's result to the following:

Corollary 6.9. Let $p$ be an odd prime, and $n$ be an integer which is not divisible by $p$. If a p-group acts freely on $\left(S^{n}\right)^{k}$ with a trivial action on homology, then $G$ has the $\Omega$-extension property.

This brings us slightly closer to answering the following question:

Question 6.10. Is there a non-abelian $p$-group which can act freely and homologically trivially on some $\left(S^{n}\right)^{k}$ where $n+1$ is not divisible by $p$ ?

Ultimately, the hope is to combine the dimension and rank restrictions in one theory that explains all the $p$-groups which can act freely on $\left(S^{n}\right)^{r}$ for any given $r$ and $n$.

\section{ACKNOWLEDGEMENTS}

I am grateful to Alejandro Adem and Jim Davis for their support during the preparation of this paper.

\section{REFERENCES}

[1] A. Adem, Z/p actions on $\left(S^{n}\right)^{k}$, Trans. A.M.S. 300 (1987), 791-809. MR 88b:57037

[2] A. Adem and J. Smith, On spaces with periodic cohomology, preprint.

[3] A. Adem and D.J. Benson, Abelian groups acting on products of spheres, Math. Z. 228 (1998), 705-712. MR 99k:57033

[4] A. Adem and W. Browder, The free rank of symmetry on $\left(S^{n}\right)^{k}$, Invent. Math. 92 (1988), 431-440. MR 89e:57034

[5] A. Adem and E. Yalçın, On some examples of group actions and group extensions, Journal of Group Theory 2 (1999), 69-79. [MR 2000b:57050]

[6] C. Allday, Elementary abelian p-group actions on lens spaces, Topology Hawaii (Honolulu, HI, 1990), 1-11, World Sci. Publishing, River Edge, NJ, 1992. MR 93e:57068

[7] K. Alzubaidy, Free actions of p-groups $(p \geq 3)$ on $S^{n} \times S^{n}$, Glasgow Math. J. 23 (1982), 97-101. MR 83i:57032

[8] J.F. Carlson, Depth and transfer maps in the cohomology of groups, Math. Z. 218 (1995), 461-468. MR 95m:20058 
[9] G. Carlsson, On the non-existence of free actions of elementary abelian groups on products of spheres, Amer. J. Math. 102 (1980), 1147-1157. MR 82a:57038

[10] G. Carlsson, On the rank of abelian groups acting freely on $\left(S^{n}\right)^{k}$, Invent. Math. 69 (1982), 393-400. MR 84e:57033

[11] A. Heller, A note on spaces with operators, Ill. J. Math. 3 (1959), 98-100. MR 21:6588

[12] G. Lewis, Free actions on $S^{n} \times S^{n}$, Trans. A.M.S. 132 (1968), 531-540. MR 37:4809

[13] B. Oliver, Free compact group actions on products of spheres, Algebraic Topology, Aarhus 1978, Lecture Notes in Math. 763, Springer-Verlag, 1979, 539-548. MR 81k:55005

[14] A. Yu. Ol'shanskii, The number of generators and orders of abelian subgroups of finite $p$ groups, Math. Notes 23 (1978), 183-185.

[15] U. Ray, Free linear actions of finite groups on products of spheres, J. Algebra 147 (1992), 456-490. MR 93d:20019

[16] T.S. Weigel, Combinatorial properties of p-central groups, preprint.

[17] T.S. Weigel, $p$-Central groups and Poincaré duality, to appear in Trans. A.M.S. CMP 98:12

[18] N. Yagita, On the dimension of spheres whose product admits a free action by a non-abelian group, Quart. J. Math. 36 (1985), 117-127. MR 86h:57041

[19] E. Yalçın, Group actions and group extensions, Trans. A.M.S. 352 (2000), no. 6, 2689-2700. CMP 2000:10

Department of Mathematics, Indiana University, Bloomington, Indiana 47405

Current address: Department of Mathematics, Bilkent University, Ankara, Turkey 06533

E-mail address: yalcine@math.mcmaster.ca 